\title{
Is tetrahydrobiopterin a therapeutic option in diabetic hypertensive patients?
}

\author{
This article was published in the following Dove Press journal: \\ Integrated Blood Pressure Control \\ 28 September 2010 \\ Number of times this article has been viewed
}

\section{Alberto Francisco Rubio- Guerra' \\ Hilda Vargas-Robles ${ }^{2}$ \\ Luz Maria Ramos-Brizuela' \\ Bruno Alfonso Escalante- \\ Acosta $^{2}$ \\ 'Metabolic Clinic, Hospital General de Ticomán SS DF, Mexico; ${ }^{2}$ Department of Molecular Biomedicine, Centro de Investigacion y de Estudios Avanzados del IPN, Mexico}

\begin{abstract}
Nitric oxide (NO) is an important regulator of vascular tone, and is also an antithrombotic, anti-inflammatory, antiproliferative, and antiatherogenic factor. Endothelial function is altered in patients with coronary artery disease, stroke, and peripheral artery disease, and endothelial dysfunction correlates with the risk factor profile for a patient. Hypertension and type 2 diabetes are risk factors for vascular disease, and are both pathologies characterized by loss of NO activity. Indeed, endothelial dysfunction is usually present in diabetic and/or hypertensive patients. Tetrahydrobiopterin is an essential cofactor for the NO synthase enzyme, and insufficiency of this cofactor leads to uncoupling of the enzyme, release of superoxide, endothelial dysfunction, progression of hypertension, and finally, proatherogenic effects. Tetrahydrobiopterin is also an important mediator of NO synthase regulation in type 2 diabetes and hypertension, and may be a rational therapeutic target to restore endothelial function and prevent vascular disease in these patients. The aim of this paper is to review the rationale for therapeutic strategies directed to biopterins as a target for vascular disease in type 2 diabetic hypertensive patients.
\end{abstract}

Keywords: tetrahydrobiopterin, endothelial dysfunction, diabetes, hypertension, oxidative stress, nitric oxide, eNOS synthase uncoupling

\section{Introduction}

The endothelium maintains the integrity of the vascular system via interaction between nitric oxide (NO) and vasoconstrictive factors. ${ }^{1}$ Endothelial dysfunction develops when the bioavailability of NO decreases, triggering a vasoconstrictive, proliferative, proinflammatory, and procoagulant condition that facilitates vascular damage. ${ }^{1,2}$

Both type 2 diabetes and hypertension increase oxidative stress and lead to endothelial dysfunction. ${ }^{1}$ Endothelial dysfunction plays a key role in the pathophysiology of atherogenesis and diabetes-associated vascular disease, and explains, at least in part, the enhanced progression of cardiovascular disease in type 2 diabetes. $^{3}$

Despite being a radical, oxygen is sparingly reactive because its two unpaired electrons are situated in different molecular orbits. However, in endothelial cells, oxygen undergoes univalent reduction to form superoxide by means of enzymes such as nicotinamide adenine dinucleotide phosphate (NADH/NADPH) oxidase. ${ }^{4}$ Vascular NADH/NADPH oxidase is active during normal metabolism, ${ }^{5}$ and sustained activation of this enzyme occurs in response to several stimuli, including angiotensin II, thrombin, platelet-derived growth factor, endothelin-1, tumor necrosis factor-alpha (TNF $\alpha$ ), hypercholesterolemia, and hyperglycemia. ${ }^{6}$ Also, some vascular flow conditions may determine regulation of NADPH oxidase, whereby laminar shear stress downregulates 
NADPH oxidase activity, whereas oscillatory shear stress induces a sustained increase in oxidase activity. ${ }^{4}$

\section{Tetrahydrobiopterin}

It was once believed that the only function of tetrahydrobiopterin (BH4) was as a cofactor for the activity of phenylalanine, tyrosine, and tryptophan hydroxylases during neurotransmitter synthesis. However, 20 years ago, when nitric oxide synthase (NOS) was characterized, BH4 was soon identified as one of its essential cofactors. ${ }^{7}$ Since this observation, $\mathrm{BH} 4$ has been implicated as a significant determinant of NO bioavailability and concomitant conduit/resistance vessel functions.

Each monomer of endothelial NOS (eNOS) has one $\mathrm{BH} 4$ binding site in the oxygenase domain and because the enzyme acts functionally as a dimer, two molecules of BH4 are incorporated into each eNOS complex. ${ }^{8}$ In the active site, BH4 stabilizes the ferrous-dioxygen complex, and the cofactor also donates electrons to the oxygenase domain, and this is the initiating step of L-arginine oxidation. ${ }^{9,10}$ If $\mathrm{BH} 4$ is reduced, electron transfer from eNOS becomes uncoupled from L-arginine oxidation, the ferrous-dioxygen complex dissociates, and the enzyme produces superoxide instead of NO.

When large amounts of reactive oxygen species (ROS) are present in the endothelial cell, electron transfer within the active site of eNOS becomes "uncoupled" from L-arginine oxidation. This process is known as eNOS uncoupling and under those conditions, electron flow through the enzyme results in reduction of molecular oxygen at the prosthetic heme site rather than formation of NO, and molecular oxygen is reduced to form superoxide, leading to endothelial dysfunction. ${ }^{11}$

Several studies have shown that when BH4 is oxidized to dihydrobiopterin (BH2), the bioavailability of $\mathrm{BH} 4$ for eNOS is reduced. This is seen when BH4 reacts with superoxide or with peroxynitrite, which leads to eNOS uncoupling and finally, to endothelial dysfunction. ${ }^{11}$ In addition, BH2 (which has no cofactor activity) may compete with BH4 for the oxygenase domain in eNOS, leading to decreased eNOS activity. ${ }^{8}$

\section{Tetrahydrobiopterin synthesis}

Biosynthesis of BH4 can occur by one of three pathways, ie, from guanosine triphosphate cyclohydrolase I (GTP-CHI) via a de novo synthetic pathway, from sepiapterin via the salvage pathway, and via recycling pathways. ${ }^{12}$

Via the de novo pathway, BH4 synthesis is initiated by the action of GTP-CHI, which represents the rate-controlling
Table I Factors that increase guanosine triphosphate cyclohydrolase I activity

\begin{tabular}{lll}
\hline Cytokines & Transcriptional factors & $\begin{array}{l}\text { Inflammatory } \\
\text { mediators }\end{array}$ \\
\hline $\begin{array}{ll}\text { Interferon } \gamma \\
\text { Tumor necrosis }\end{array}$ & $\begin{array}{l}\text { Nuclear factor } \mathrm{\kappa B} \\
\text { Signal transducer } \\
\text { factor } \alpha\end{array}$ & $\begin{array}{l}\text { and activator } \\
\text { of transcription } \mathrm{I} / 3\end{array}$ \\
\hline
\end{tabular}

enzyme and initiates GTP degradation to 7,8 dihydroneopterin triphosphate, which is converted to 6-pyruvoyl-tetrahydropterin by the 6-6-pyruvoyl synthase enzyme. Finally, the 6-pyruvoyltetrahydropterin is reduced to BH4 by NADPH-dependent sepiapterin reductase (Figure 1).

Via the salvage pathway, sepiapterin is metabolized to 7,8-BH2 and finally, to BH4 by two NADPH-dependent enzymes, ie, sepiapterin reductase and dehydrofolate reductase (Figure 1). However, sepiapterin is not a physiologic metabolite in humans or animals. ${ }^{9}$

In the recycling pathway, BH4 is generated differently, depending on the enzymes involved. When aromatic aminohydrolases are present, $\mathrm{BH} 4$ is regenerated from BH $4 \alpha$-carbinolamine in a two-step process, whereby pterin- $4 \alpha$ carbinolamine dehydratase produces the quinonoid $\mathrm{BH} 2$ intermediate, which is subsequently reduced by 6,7-dihydropteridine reductase. When NOS is involved, BH4 is transformed to a $\mathrm{BH} 3 . \mathrm{H}+$ radical, which is subsequently reduced in the next catalytic cycle by electron transfer from eNOS flavins ${ }^{9}$ (Figure 1).

\section{Regulation of tetrahydrobiopterin}

As mentioned in the previous section, the GTP-CH enzyme is critical for BH4 synthesis, and several studies have demonstrated that vascular agonists may increase $\mathrm{BH} 4$ production through GTP-CH mRNA induction. Incubation of endothelial cells with phenylalanine increases mRNA levels for GTP-CH by 2.2 -fold after 6 hours, ${ }^{13}$ and interestingly, similar concentrations of phenylalanine increase $\mathrm{BH} 4$ levels in bovine coronary endothelial cells by $16 \% .{ }^{14}$ Insulin increases mRNA for GTP-CH, which enhances endothelial BH4 through the activation of the de novo pathway. ${ }^{15}$ Addition of $17 \beta$-estradiol to culture medium elevated both mRNA levels for CGT-CH and intracellular BH4 levels in brain microvascular endothelial cells. ${ }^{16}$ Inflammatory cytokines, such as interleukin (IL)-1 $\beta$, interferon- $\gamma$ or TNF $\alpha$, increase BH4 synthesis, probably through the effect of increasing mRNA for GTP-CH. ${ }^{17}$ Finally, statins and cyclosporine can increase BH4 synthesis and GTP-CH mRNA in cultures of endothelial cells. ${ }^{14,18}$ 


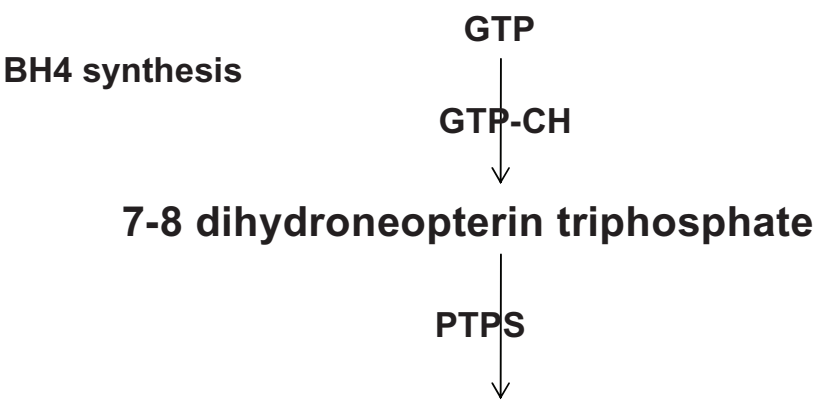

6 Pyruvoyletetrahydrobiopterin

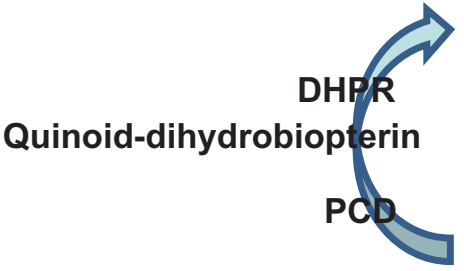

\section{Tetrahydrobiopterin $4^{\alpha}$-carbinolamine}

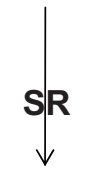

$\mathrm{BH} 4$<smiles>CCCCCCCCCC</smiles>

$\downarrow$

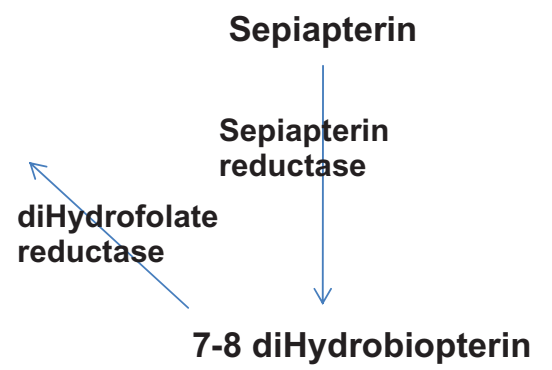

7-8 diHydrobiopterin

Figure I Tetrahydrobioterin synthesis.

Abbreviation: BH4, tetrahydrobiopterin; GTP-CH, GTP cyclohydrolase; PTPS, pyruvoyl tetrahydrobiopterin synthase; SR, sepiapterin reductase; DHPR, dihydrofolate reductase.

On the other hand, several studies have demonstrated that some vascular agonists may decrease $\mathrm{BH} 4$ production through GTP-CH mRNA downregulation. Johns et al found that dexamethasone decreases the expression of GTP-CH mRNA and BH4 levels in endothelial cells. ${ }^{19}$ Similarly, inflammatory cytokines, such as IL-4, IL-10, and transforming growth factor-beta, decrease BH4 synthesis, probably through GTP-CH mRNA downregulation. ${ }^{20}$

In addition to regulation of $\mathrm{BH} 4$ synthesis through these already described mRNA mechanisms, there are two other conditions where changes in $\mathrm{BH} 4$ bioavailability have been demonstrated. It has recently been reported that increasing concentrations of L-arginine raised intracellular levels of BH4 production in cultured bovine coronary endothelial cells. This effect is specific for the L-isomer and is not affected by the use of arginase inhibitors. ${ }^{14}$ This in vitro observation was further supported by in vivo studies in which dietary supplementation of L-arginine increased $\mathrm{BH} 4$ availability in coronary endothelial cells from control and diabetic rats. ${ }^{21}$ In light of the reducing properties of $\mathrm{BH} 4$, the possibility that oxidative stress may affect $\mathrm{BH} 4$ availability arises. Indeed, the use of peroxynitrite in intact arteries demonstrated that oxidative stress is associated with $\mathrm{BH} 4$ reduction. ${ }^{22}$ Moreover, the hypothesis that $\mathrm{BH} 4$ acts as a reducing agent is further supported by the demonstration that vitamin $\mathrm{C}$ stabilizes $\mathrm{BH} 4$ in cultured endothelial human cells, through a mechanism not related to the interaction of vitamin $\mathrm{C}$ and superoxide anions. ${ }^{23}$

\section{Interaction between tetrahydrobiopterin and nitric oxide synthase}

$\mathrm{NO}$ is synthesized from L-arginine by NOS. Molecular cloning has identified three distinct NOS isoforms, ${ }^{24}$ two of which are expressed constitutively in neurons and vascular endothelial cells, and are activated by increased intracellular calcium levels. The expression of the third isoform is induced in a calcium-independent fashion by several agonists. All NOS isoforms catalyze the reaction of $1.5 \mathrm{~mol} \mathrm{NADPH}+1 \mathrm{~mol}$ L-arginine to $1 \mathrm{~mol}$ citrulline $+1 \mathrm{~mol} \mathrm{NO}+1.5 \mathrm{NADP}+$. This reaction is fully dependent on BH4. NOS contains a prosthetic heme group catalyzing the reductive activation of molecular oxygen which is required for L-arginine oxidation, as well as tightly bound flavins shuttling NADPH-derived electrons to the heme (Figure 2). ${ }^{25}$ In this reaction, heme $\mathrm{Fe}^{3+}$ is reduced by flavin mononucleotide to heme $\mathrm{Fe}^{2+}$, which binds molecular oxygen to form an unstable dioxygen complex (heme-Fe-O-O) that, in the presence of $\mathrm{BH} 4$, is converted to heme- $\mathrm{Fe}^{3+}-\mathrm{O}-\mathrm{OH}$, then converted to heme- $\mathrm{Fe}^{5+}=\mathrm{O}$, to participate in the release of $\mathrm{NO}$ (Figure 2). BH4 in this reaction participates in the following events: $\mathrm{BH} 4$ binding to NOS 


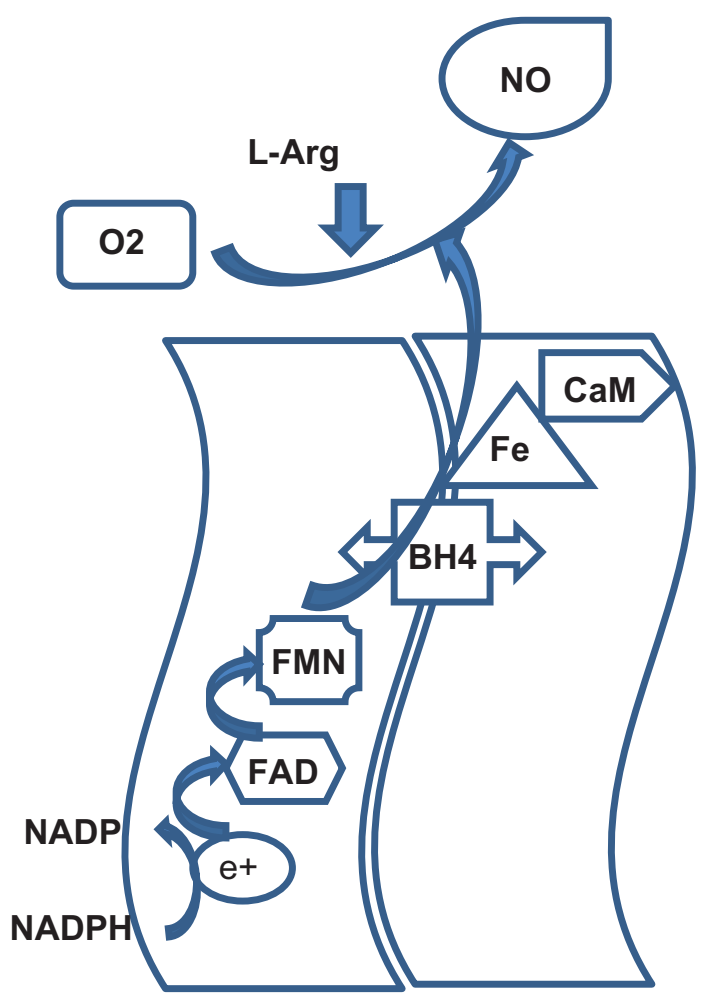

\section{Coupled NOS}

Figure 2 Interaction between tetrahydrobiopterin and nitric oxide synthesis.

stabilizes loose dimer conformation; ${ }^{26} \mathrm{BH} 4$ binding to NOS enhances L-arginine binding to heme and inhibits superoxide release from the heme oxygenase domain of the enzyme. ${ }^{27}$

NO synthesis by eNOS can be affected by different stimuli, ie, signaling molecules (integrated into caveolae), phosphorylation (by Akt) at serine 177 residues in response to shear stress or hormones (such as estrogens or insulin) activating the enzyme, whereas phosphorylation at threonine 459 (by protein kinase C) decreases the activation of eNOS. Dephosphorylation of threonine 459 in response to bradykinin activates eNOS. ${ }^{8}$

Deficient BH4 levels in several in vitro and in vivo animal models have correlated with low NO production in vascular tissue. ${ }^{28}$ Recent studies suggest that disruption of the zinc-thiolate complex at the dimer interface, close to the BH4 binding site, leads to loss in BH4 from the binding site, enzymatic uncoupling, and destabilization of eNOS dimers. ${ }^{29}$

The data suggest that eNOS uncoupling and increased nitrosylation of eNOS, decreased expression of GTP-CHI and sepiapterin reductase, and subsequent reduced $\mathrm{BH} 4$ bioavailability, may be important contributors to endothelial dysfunction and its consequences.

NO produced by endothelial cells protects blood vessels from thrombosis, and has antiatherosclerotic activity. Enhanced

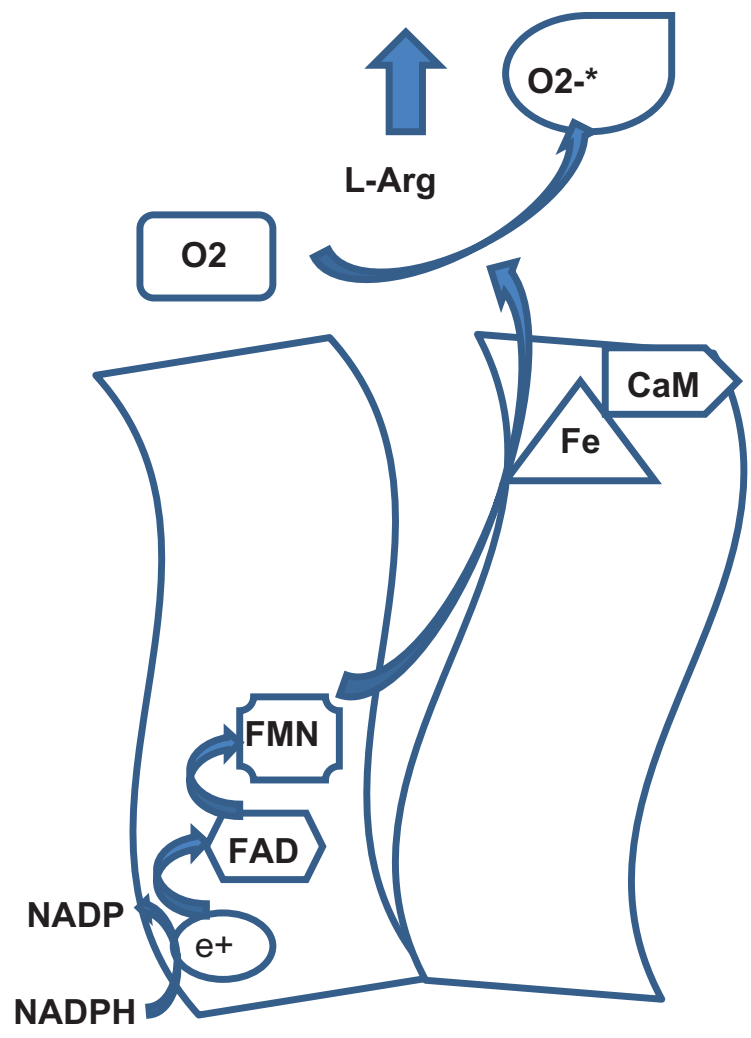

Uncoupled NOS

NO bioactivity reduces atherosclerosis progression through multiple mechanisms (Table 2). All major risk factors for atherosclerosis are associated with impairment of $\mathrm{NO}$ activity. ${ }^{15}$

Superoxide generation has been implicated in a variety of experimental and clinical vascular disease states, including diabetes, cigarette smoking, hypertension, chronic nitrate tolerance, and overt atherosclerosis, not only because of the increased production of ROS but also because of the reduced formation of the protective molecule $\mathrm{NO}^{31}$

\section{Tetrahydrobiopterin in disease}

In the absence of enough BH4, instead of oxidizing L-arginine, eNOS reduces molecular oxygen to superoxide, leading to endothelial dysfunction. ${ }^{32}$ When NO reacts with superoxide, it loses its vasodilatory, antiatherogenic, antithrombotic, antiinflammatory, and antiproliferative effects. ${ }^{33}$

Table 2 Antiatherosclerotic effects of nitric oxide

- Decreases endothelial permeability

- Inhibition of low-density lipoprotein oxidation

- Reduces influx of lipoproteins into the vascular wall

- Inhibition of proliferation of vascular smooth muscle cells

- Prevention of leukocyte adhesion to vascular endothelium

- Inhibition of leukocyte migration into the vascular wall 
BH4 prevents peroxynitrite-induced nitration of proteins, and the prevention of nitration of tyrosine residues in cells stimulated by proinflammatory cytokines avoids the affectation of proteins involved in energy production, fatty acid metabolism, apoptosis, and oxidative stress induced by peroxynitrite. $^{34}$

Experimental evidence reveals that altered glucose metabolism results in low $\mathrm{BH} 4$ bioavailability, and also that $\mathrm{BH} 4$ levels are reduced in diabetic rats. Interestingly, dietary supplementation with BH4 in insulin-resistant rats prevents impaired endothelial-dependent vasodilatation. ${ }^{12}$

The main cause of impaired endothelium-dependent relaxation in insulin-resistant rats is abnormal metabolism of BH4. The mechanism seems to be an imbalance between $\mathrm{NO}$ and superoxide anion. ${ }^{32} \mathrm{NO}$ regulates vascular tone and blood pressure, and a reduction in NO bioavailability increases vascular tone and raises blood pressure, ${ }^{4}$ so BH4 may be involucrate in the maintenance of blood pressure and a decrease in its bioavailability may play a role in the pathways that lead to hypertension. ${ }^{12}$

\section{Therapeutic considerations $\mathrm{BH} 4$ as a therapeutic agent}

Several in vivo studies in animals and in humans have shown the beneficial effect of $\mathrm{BH} 4$ on endothelial dysfunction and its vasoprotective properties. Therefore, enhancing BH4 synthesis or bioavailability in endothelial cells may be a good strategy for the prevention and treatment of cardiovascular disease, especially in high-risk patients, such as hypertensive and diabetic subjects. ${ }^{34}$

When BH4 is administrated in healthy volunteers, the bioavailability of NO is increased. Several clinical trials have evaluated the use of $\mathrm{BH} 4$ for the management of endothelial dysfunction, and it has been shown that this cofactor increases acetylcholine-induced vasodilatation in coronary arteries in patients with coronary heart disease. ${ }^{35}$

The administration of BH4 improves forearm circulation in smokers, and diabetic or hypercholesterolemic patients, then, actions that lead to improve $\mathrm{BH} 4$ availability may be effective in restoring NO-mediated endothelial function and limiting vascular disease progression in several conditions, such as atherosclerosis, diabetes, and hypertension. ${ }^{36}$ Indeed, infusion of BH4 improves the endothelial-dependent vasodilation response to acetylcholine but not the endothelium-independent vasodilation response to nitroprusside. In patients with type 2 diabetes, the cofactor has no effect in control subjects. ${ }^{32}$

In healthy subjects, impairment of endothelial function induced by an oral glucose challenge was reversed by the active cofactor $\mathrm{BH} 4$, but not by an inactive stereoisomer. ${ }^{37}$ In patients with type 2 diabetes, infusion of $\mathrm{BH} 4$ corrects endothelial dysfunction via an NO-dependent pathway. ${ }^{12}$

The administration of BH4 to spontaneously hypertensive rats is associated with improvement of endothelial function and prevention of hypertension and ventricular hypertrophy. ${ }^{12}$

Porkert et al found that oral administration of BH4 $400 \mathrm{mg}$ had a significant antihypertensive effect in poorly controlled hypertensive patients. ${ }^{38}$ They also found an improvement of endothelial function in this study, and the beneficial effect of BH4 was maintained during the eight-week study duration without tachyphylaxis. However, only patients receiving the BH4 dose of $400 \mathrm{mg}$ or higher responded to the biopterin, whereas patients at a daily dose of $200 \mathrm{mg}$ did not achieve a significant reduction in blood pressure.

Settergren et al showed that the administration of L-arginine and BH4 improves endothelial function and reduces endothelial dysfunction induced by ischemia-reperfusion in type 2 diabetic patients with coronary artery disease.$^{39}$ However, both agents were given in combination, so it was not possible to evaluate if administration of each agent alone would demonstrate the same results or if the combination is necessary. It is important to say that L-arginine as monotherapy was not effective, and perhaps harmful, in patients with intermittent claudication and peripheral artery disease. ${ }^{40}$

$\mathrm{BH} 4$ has shown efficacy in the treatment of erectile dysfunction. Sommer et al found that BH4 administration led to a significant increase in duration of penile rigidity, making BH4 suitable as a treatment for erectile dysfunction. ${ }^{41}$

Oral administration of BH4 slows the progression of atherosclerosis and reduces the expression of NADPH oxidase and inflammatory factors in hypercholesterolemic apolipoprotein E-knockout mice. ${ }^{42}$

\section{Other pharmacologic interventions to restore $\mathrm{BH} 4$ bioavailability and eNOS function}

\section{Nitrates}

Nitrates (isosorbide, nitroglycerin) have been used as NO donors because they release NO. ${ }^{43}$ Unfortunately, chronic administration of nitroglycerin has been associated with vascular production of superoxide and poor clinical benefits. ${ }^{12,44} \mathrm{~A}$ long-acting nitrate, pentaerythritol tetranitrate, may confer a better long-term cardiovascular prognosis. ${ }^{44}$

\section{L-arginine}

Several clinical studies have shown vasodilatation and enhanced NO production after administration of L-arginine. 
Drexler et al (cited by Tenenbaum et $\mathrm{al}^{45}$ ) showed that L-arginine enhanced the blood flow response to acetylcholine in patients with coronary artery disease but not in controls. Since then, there have been many studies with L-arginine in healthy human subjects and in patients with various cardiovascular conditions. ${ }^{45}$

In contrast, there have been several relatively small clinical studies with experimental endpoints that failed to show beneficial effects of L-arginine on vascular function. The evidence suggests that there may be subgroups of patients whose vascular function is improved by L-arginine supplementation, and other subgroups of patients who do not respond to this intervention. Because patients on "optimized medical treatment" and those with advanced coronary stenoses showed less effect, and Larginine was more effective when early changes in vascular function were chosen as endpoints. It is possible that this amino acid may have a role as a nutraceutical agent (daily doses below 2 to 3 $\mathrm{g}$ /day appear to be without beneficial effect) in the modification of functional impairment and in the prevention of vascular disease, but not as a therapy to reverse manifest atherosclerosis. ${ }^{45,46}$ In animal models, long-term administration of L-arginine inhibits the formation of atherosclerotic plaques. ${ }^{44}$

\section{Folic acid}

Because folic acid participates in the $\mathrm{BH} 4$ recycling pathway, several studies have been performed to investigate its use for the treatment of cardiovascular disease. Some results had shown that this agent may restore endothelial function in diabetic patients, as well as in hypercholesterolemic subjects, and that it reduces atherosclerotic plaques in mice. ${ }^{44,46}$ Unfortunately, those results have not been confirmed by recent clinical trials. ${ }^{47}$

\section{Ascorbate}

Ascorbate increases $\mathrm{BH} 4$ in endothelial cells via prevention of oxidation of this biopterin, without an effect on its synthesis, and may explain the effect of ascorbate on blood pressure in hypertensive patients. ${ }^{33}$

\section{Sepiapterin}

Because sepiapterin is a precursor in the alternative BH4 synthesis pathway, it has been proposed as a therapeutic approach for endothelial dysfunction. ${ }^{12}$ Indeed, acute studies have shown that administration of sepiapterin restores endothelial function. ${ }^{44}$ However, because sepiapterin competes with BH4 for the oxygenase domain in eNOS, higher doses of this precursor may increase superoxide production and decrease NO bioavailability. ${ }^{12,44}$ There are no studies that have evaluated the long-term effects of sepiapterin in vascular disease. This agent did not increase BH4 levels, but decreased $\mathrm{BH} 2$ concentrations, ${ }^{44}$ and a recent study shows that the $\mathrm{BH} 4 / \mathrm{BH} 2$ ratio may be even more important than absolute BH4 levels for eNOS functioning. ${ }^{49}$

\section{Statins}

Statins (3-hydroxy-3-methylglutaryl coenzyme A reductase inhibitors) are used in the management of dyslipidemia. These drugs have cholesterol-independent effects, may upregulate NO expression, and there is evidence that statins modulate atherogenesis, plaque rupture, and thrombosis. ${ }^{50}$

Hattori et al found that statins elevate GTP-CH mRNA. Because GTP-CH is the rate-limiting step in the synthesis of BH4, statins elevate levels of this biopterin in vascular endothelial cells, and they also enhance eNOS expression and increase the ratio of $\mathrm{BH} 4 / \mathrm{BH} 2 .^{51}$

Because statins enhance BH4 synthesis, increase NO production, and prevent relative shortages of $\mathrm{BH} 4$, they also inhibit vascular NADPH oxidase and reduce oxidative stress, ${ }^{44}$ thereby normalizing endothelial function. In humans, statins not only reduce cardiovascular morbidity and mortality, but also reduce progression of coronary atherosclerosis in patients with coronary heart disease. ${ }^{52}$

\section{Antagonism of the renin-angiotensin- aldosterone system}

Angiotensin II activates NADPH oxidase via $\mathrm{AT}_{1}$ receptor activation in vascular cells. However, NADPH oxidase is probably not the only source of ROS stimulated by angiotensin II. As we have reviewed, one consequence of increased superoxide production in response to angiotensin II is inactivation of $\mathrm{NO}$ and resulting endothelial dysfunction, which is one of the earliest steps in the atherosclerotic process. ${ }^{52}$

Several studies have shown that inhibition of $\mathrm{AT}_{1}$ receptor activation by $\mathrm{AT}_{1}$ receptor antagonists or angiotensinconverting enzyme (ACE) inhibitors normalizes oxidative stress and improves endothelial dysfunction, and ACE inhibitors are known to retard the progression of atherosclerosis and heart failure. ${ }^{53}$ Both $\mathrm{AT}_{1}$ receptor antagonists and ACE inhibitors provide an equivalent beneficial effect on risk of death and cardiovascular events. ${ }^{54}$

Like angiotensin II, aldosterone may also promote endothelial dysfunction and vascular disease. Imanishi et al found that eplerenone, an aldosterone antagonist, alone or combined with enalapril, reduces NADPH activity, and elevates both BH4 levels and NO bioavailability in endothelial cells of hyperlipidemic rabbits. ${ }^{55}$ 
Aliskiren, a direct renin inhibitor, as monotherapy or in combination with an $\mathrm{AT}_{1}$ receptor antagonist, also decreases NADPH oxidase activity, increases BH4 levels, and enhances NO bioavailability. Aliskiren also has an antiatherosclerotic effect. $^{56}$

\section{Conclusion}

BH4 availability is a critical determinant of eNOS regulation in atherosclerosis and is a rational therapeutic target to restore NO-mediated endothelial function and reduce disease progression in high-risk patients.

Strategies aimed at increasing BH4 biosynthesis or levels, reducing $\mathrm{BH} 4$ oxidation, or enhancing $\mathrm{BH} 4$ regeneration seem to be useful and safe therapeutic options for the prevention of macrovascular complications in patients at high risk for cardiovascular complications, in particular patients with type 2 diabetes and/or hypertension.

\section{Disclosure}

AFRG is a research fellow, and received a grant from Consejo Nacional de Ciencia y Tecnologia (CONACYT) Mexico in support of this research.

\section{References}

1. Ghanem FA, Movahed A. Inflammation in high blood pressure: A clinical perspective. J Am Soc Hypertens. 2007;1:113-119.

2. Rubio-Guerra AF, Vargas-Robles H, Vargas-Ayala G, Rodríguez-Lopez L, Escalante-Acosta BA. The effect of trandolapril and its fixed-dose combination with verapamil on circulating adhesion molecules levels in hypertensive patients with type 2 diabetes. Clin Exp Hypertens. 2008;30:682-688.

3. Rubio AF, Vargas H, Maceda A, Lozano JJ, Escalante BA. Correlation between the levels of circulating adhesion molecules and atherosclerosis in type-2 diabetic normotensive patients. Cell Adh Migr. 2009;3:369-372.

4. Madamanchi NR, Vendrov A, Runge MS. Oxidative stress and vascular disease. Arterioscler Thromb Vasc Biol. 2005;25:29-38.

5. Griendling KK, Sorescu D, Ushio-Fukai M. NAD(P)H oxidase: Role in cardiovascular biology and disease. Circ Res. 2000;86:494-501.

6. Kim J, Montagnami M, Kon K, Quon MJ. Reciprocal relations between insulin resistance and endothelial dysfunction. Circulation. 2006;113:1888-1904.

7. Kwon NS, Nathan CF, Stuerhr DJ. Reduced biopterin as a cofactor in the generation of nitrogen oxides by murine macrophages. $\mathrm{J} \mathrm{Biol} \mathrm{Chem.}$ 1989;264:20496-20501.

8. Alp NJ, Channon KM. Regulation of endothelial nitric oxide synthase by tetrahydrobiopterin in vascular disease. Arterioscler Thromb Vasc Biol. 2005;24:413-420.

9. Gross S, Jones S, Hattori CL, Raman CS. Tetrahydrobiopterin: An essential cofactor of nitric oxide synthase with an elusive role. In: Ignarro LJ, editor. Nitric Oxide Biology and Pathobiology. New York, NY: Academic Press; 2000.

10. Hurshman AR, Krebs C, Edmondson D, Huynh BH, Marletta MA. Formation of a pterin radical in the reaction of the heme domain of inducible nitric oxide synthase with oxygen. Biochemistry. 1999; 38:15689-15696.
11. Bauersachs J, Widder JD. Tetrahydrobiopterin, endothelial nitric oxide synthase, and mitochondrial function in the heart. Hypertension. 2009;53:907-908.

12. Schmidt TS, Alp NJ. Mechanisms for the role of tetrahydrobiopterin in endothelial function and vascular disease. Clin Sci. 2007;113:47-63.

13. Gesierich A, Niroomand F, Tiefenbavher CP. Role of human GTP cyclohydrolase I and its regulatory protein in tetrahydrobiopterin metabolism. Basic Res Cardiol. 2002;98:69-75.

14. Wu G, Kelly KA, Hatakeyama K, Meininger CJ. Regulation of endothelial tetrahydrobiopterin synthesis by l-arginine. In: Blau N, Thony B, editors. Pterins, Folates and Neurotransmitters in Molecular Medicine. Heilbronn: SPS Verlagsgesellschaft mbH; 2004.

15. Ishii M, Shimizu S, Nagai T, Shiota K, Kiruchi Y, Yamamoto T. Stimulation of tetrahydrobiopterin synthesis by insulin: Possible involvement of phosphatidylinositol 3-kinase. Int J Biochem Cell Biol. 2001;33:65-73.

16. Shiota K, Ishii M, Yamamoto T, Shimizu S, Kiuchi Y. Stimulation of tetrahydrobiopterin synthesis by $17 \beta$-estradiol in brain microvascular endothelial cells. Pteridines. 2000;11:129-132.

17. Rosenkrantz-Weiss P, Sessa WC, Milstein S, Kaufman S, Watson CA, Pober JS. Regulation of nitric oxide synthesis by proinflammatory cytokines in human umbilical vein endothelial cells. J Clin Invest. 1994;93:2236-2243.

18. Ishii M, Shimizu S, Shiota K, Yamamoto S, Kiuchi Y, Yamamoto T. Stimulation of tetrahydrobiopterin synthesis by cyclosporin A during lipopolysaccharide treatment in vascular endothelial cells. Pteridines. 2002;13:89-93.

19. Johns DG, Dorrance AM, Tramontini NL, Webb RC. Glucocorticoids inhibit tetrahydrobiopterin-dependent endothelial function. Exp Biol Med. 2001;226:27-31.

20. Schoedon G, Scheemann M, Blau N, Edgell CJS, Chafner A. Modulation of human endothelial cell tetrahydrobiopterin synthesis by activating and deactivating cytokines: New perspectives on endothelium-derived relaxing factor. Biochem Biophys Res Comm. 1993;196:1343-1348.

21. Kohli R, Meininger CJ, Haynes TE, Yan W, Self JT, Wu G. Dietary L-arginine supplementation enhances endothelial nitric oxide synthesis in streptozotocin-induced diabetic rats. $J$ Nutr. 2004;134:600-609.

22. Laursen JB, Somers M, Kurz S, et al. Endothelial regulation of vasomotion in ApoE-deficient mice. Implications for interactions between peroxynitrite and tetrahydrobiopterin. Circulation. 2001; 103:1282-1288.

23. Baker H, Milstien S, Katusik ZS. Effect of vitamin C on the availability of tetrahydrobiopterin in human endothelial cells. $J$ Cardiovasc Pharmacol. 2001;37:333-338.

24. Knowles RG, Moncada S. Mammalian nitric oxide synthase. Biochem J. 1994;298:249-254.

25. Cosentino F, Luscher TF. Tetrahydrobiopterin and endothelial nitric synthase activity. Cardiovasc Res. 1999;43:274-278.

26. Whitsett J, Martasek P, Zhao H, et al. Endothelial cell superoxide anion radical generation is not dependent on endothelial nitric oxide synthaseserine 1179 phosphorylation and endothelial nitric oxide dimer/ monomer distribution. Free Rad Biol Med. 2006;40:2056-2058.

27. Vazquez-Vivar J. Tetrahydrobiopterin, superoxide and vascular dysfunction. Free Rad Biol Med. 2009;47:1108-1119.

28. Crabtree MJ, Smith CI, Lam G, Goligorsky MS, Gross SS. Ratio of 5,6,7,8-tetrahydrobiopterin to 7,8-dihydrobiopterin in endothelial cells determines glucose-elicited changes in NO vs superoxide production by eNOS. Am J Physiol Heart Circ Physiol. 2008;294:H1530-H1540.

29. Zou MH, Shi C, Cohen RA. Oxidation of the zinc-thiolate complex and uncoupling of endothelial nitric oxide synthase by peroxynitrite. J Clin Invest. 2002;109:817-826.

30. Yang Y, Huang A, Kaley G, Sun D. eNOS uncoupling and endothelial dysfunction in aged vessels. Am J Physiol Heart Circ Physiol. 2009;297: H1829-H1836.

31. Stuehr D, Pou S, Rosen GM. Oxygen reduction by nitric-oxide synthases. J Biol Chem. 2001;276:14533-14536. 
32. Heitzer T, Krohn K, Albers S, Meinertz T. Tetrahydrobiopterin improves endothelial vasodilation by increasing nitric oxide activity in patients with Type II diabetes mellitus. Diabetologia. 2000;43:1435-1438.

33. Landmesser U, Dikalov S, Price SR, et al. Oxidation of tetrahydrobiopterin leads to uncoupling of endothelial cell nitric oxide synthase in hypertension. J Clin Invest. 2003;111:1201-1209.

34. Katusic ZS, d'Uscio L, Nath KA. Vascular protection by tetrahydrobiopterin: Progress and therapeutic prospects. Trends Pharmacol Sci. 2009;30:48-54.

35. Artunc F, Essig M, Artunc N, et al. Effects of tetrahydrobiopterin on nitric oxide bioavailability and renal hemodynamics in healthy volunteers. J Nephrol. 2008;21:850-860.

36. Alp NJ, McAteer MA, Khoo J, Choudhury RP, Channon KM. Increased endothelial tetrahydrobiopterin synthesis by targeted transgenic GTPcyclohydrolase I overexpression reduces endothelial dysfunction and atherosclerosis in ApoE-knockout mice. Arterioscler Thromb Vasc Biol. 2004;24:445-450.

37. Ihlemann N, Rask-Madsen $\mathrm{CH}$, et al. Tetrahydrobiopterin restores endothelial dysfunction induced by an oral glucose challenge in healthy subjects. Am J Physiol. 2003;285:H875-H882.

38. Porkert M, Sher S, Reddy U, et al. Tetrahydrobiopterin: A novel antihypertensive therapy. J Hum Hypertens. 2008;22:401- 407.

39. Settergren M, Böhm F, Malmström RE, Channon KM, Pernow J. L-arginine and tetrahydrobiopterin protects against ischemia/reperfusioninduced endothelial dysfunction in patients with type 2 diabetes mellitus and coronary artery disease. Atherosclerosis. 2009;204:73-78.

40. Wilson AM, Harada R, Nair N, Balasubramanian N, Cooke JP. L-arginine supplementation in peripheral arterial disease: No benefit and possible harm. Circulation. 2007;116:188-195.

41. Sommer F, Klotz T, Steineritz D, Bloch W. Evaluation of tetrahydrobiopterin as a potential therapeutic agent to treat erectile dysfunction. Asian J Androl. 2006;8:159-167.

42. Hattori Y, Hattori S, Wang X, Satoh H, Nakanishi N, Kasai K. Oral administration of tetrahydrobiopterin slows the progression of atherosclerosis in apolipoprotein E-knockout mice. Arterioscler Thromb Vasc Biol. 2007;27:865-870.

43. Rubio AF, Vargas G, Lozano JJ, Narvaez JL, Rodriguez L. Comparison between isosorbide dinitrate aerosol and nifedipine in the management of hypertensive emergencies. J Hum Hypertens. 1999;13:473-476.

44. Li H, Förstermann U. Prevention of atherosclerosis by interference with the vascular nitric oxide system. Curr Pharm Des. 2009; $15: 3133-3145$.
45. Tenenbaum A, Fisman EZ, Motro M. L-arginine: Rediscovery in progress. Cardiology. 1998;90:153-159.

46. Boger RH. The pharmacodynamics of L-arginine. J Nutr. 2007;137: S1650-S1655.

47. Stroes ESG, van Faassen EE, Yo M, et al. Folic acid reverts dysfunction of endothelial nitric oxide synthase. Circ Res. 2000;86:1129-1134.

48. The Heart Outcomes Prevention Evaluation (HOPE) 2 Investigators. Homocysteine lowering with folic acid and B vitamins in vascular disease. N Engl J Med. 2006;354:1567-1577.

49. Alp NJ, Mussa S, Khoo J, et al. Tetrahydrobiopterin-dependent preservation of nitric oxide-mediated endothelial function in diabetes by targeted transgenic GTP-cyclohydrolase I overexpression. J Clin Invest. 2003; 112:725-735.

50. Laufs U, La Fata V, Plutzky J, Liao JK. Upregulation of endothelial nitric oxide synthase by HMG CoA reductase inhibitors. Circulation. 1998; 97:1129-1135.

51. Hattori Y, Nakanishi N, Akimoto K, Yoshida M, Kasai K. HMG-CoA reductase inhibitor increases GTP cyclohydrolase I mRNA and tetrahydrobiopterin in vascular endothelial cells. Arterioscler Thromb Vasc Biol. 2003;23:176-182.

52. Nissen SE, Tuzcu EM, Schoenhagen P, et al. Effect of intensive compared with moderate lipid-lowering therapy on progression of coronary atherosclerosis: A randomized controlled trial. JAMA. 2004;291:1071-1080.

53. Nickening G, Harrison DG. The AT1-type angiotensin receptor in oxidative stress and atherogenesis. Part 1. Circulation. 2002;105: 393-396.

54. David B, Matchar DB, McCrory DC, et al. Systematic review: Comparative effectiveness of angiotensin-converting enzyme inhibitors and angiotensin II receptor blockers for treating essential hypertension. Ann Intern Med. 2008;148:16-29.

55. Imanishi $\mathrm{T}$, Ikejima $\mathrm{H}$, Tsujioka $\mathrm{H}$, et al. Addition of eplerenone to an angiotensin-converting enzyme inhibitor effectively improves nitric oxide bioavailability. Hypertension. 2008;51:734-741.

56. Imanishi $\mathrm{T}$, Tsujioka $\mathrm{H}$, Ikejima $\mathrm{H}$, et al. Renin inhibitor aliskiren improves impaired nitric oxide bioavailability and protects against atherosclerotic changes. Hypertension. 2008;52:563-572.
Integrated Blood Pressure Control

\section{Publish your work in this journal}

Integrated Blood Pressure Control is an international, peer-reviewed open-access journal focusing on the integrated approach to managing hypertension and risk reduction. Treating the patient and comorbidities together with diet and lifestyle modification and optimizing healthcare resources through a multidisciplinary team approach constitute key

\section{Dovepress}

features of the journal. This journal is indexed on American Chemical Society's Chemical Abstracts Service (CAS). The manuscript management system is completely online and includes a very quick and fair peerreview system, which is all easy to use. Visit http://www.dovepress.com/ testimonials.php to read real quotes from published authors. 